\title{
Three Double Degenerate Candidates
}

\author{
Diana Foss \\ Steward Observatory, University of Arizona \\ Tucson, AZ, 85721 USA
}

This poster reports the results of a search for variable radial velocities in 29 DA white dwarfs. The survey was sensitive to periods between $1^{\mathrm{h}}$ and $66^{\mathrm{d}}$, although non-ideal sampling limited the longest practically detectable period to $2 \mathrm{~d}$. Three stars were discovered to have radial velocity shifts at above the $3 \sigma$ level. The discovery of these stars, along with that of Saffer, et al. (1988) can put only a lower limit on the space density of close binary white dwarfs, as this survey was less than $100 \%$ efficient in detecting radial velocity variations, and its efficiency depended strongly on period.

\section{Introduction}

Several authors (Paczynski 1985, Webbink 1984, Iben and Tutukov 1984) have proposed close pairs of degenerate stars as the progenitors of Type Ia supernovae (SNela), and subsequent calculations of the merger of such systems by Iben and Tutukov $(1984,1987)$, Arnett, Branch and Wheeler (1985), and Tornambe and Matteuchi (1987), among many others, have produced model spectra and light curves in good agreement with observations of real SNeIa. This theoretical framework has motivated workers to search for duplicity in supposedly single white dwarfs. Greenstein (1986) has found a handful of wide pairs of white dwarfs, but the only major systematic search until now, that of Robinson and Shafter (1987), failed to find any binaries. They concluded that the fraction of all supposedly single white dwarfs that are really double with periods between $30^{\mathrm{s}}$ and $3^{\mathrm{h}}$ is less than $1 / 20$ with $90 \%$ probability. Nevertheless, Saffer, et al. (1988) have discovered that WD 0135-052 (EG 11, L 870-2), a popular spectrophotometric standard, is in fact a close, detached pair of DA white dwarfs. The present search was motivated by the lack of systematic observations sensitive to periods longer than three hours.

\section{Observations}

The observations were carried out using the $1.5 \mathrm{~m}$ telescope at Palomar Observatory on the nights of 19-21 June and 30 October-2 November 1985 and 23-27 February 1986. The instrument used was the $1.5 \mathrm{~m}$ 's CCD spectrograph, using a $800 \times 816$ pixel TI CCD, and a 1200 line grating in first order. The observations covered a wavelength range between 4200 to $5100 \AA$ and had a resolution of $240 \mathrm{~km} / \mathrm{sec}$. Forty-two bright white dwarfs were observed, 29 of which had spectra good enough to fit. All but 11 of these were subsequently observed by Robinson and Shafter, but the observations reported here were spaced such that much longer periods were sampled. The stars, listed in Table 1, were chosen, on the basis of their brightness, from the McCook and Sion (1984) catalog of spectroscopically identified white dwarfs. Each observation was bracketed by 


\section{Table 1}

\begin{tabular}{|c|c|c|c|c|c|}
\hline Star & $\begin{array}{c}\left\langle\sigma_{\mathrm{v}}\right\rangle \\
(\mathrm{km} / \mathrm{sec})\end{array}$ & $\begin{array}{c}\Delta t \\
(\mathrm{hr})\end{array}$ & Star & $\begin{array}{c}\left\langle\sigma_{\mathrm{v}}\right\rangle \\
(\mathrm{km} / \mathrm{sec})\end{array}$ & $\begin{array}{c}\Delta t \\
(\mathrm{hr})\end{array}$ \\
\hline WD $0004+330$ & 76.42 & 45.92 & WD 1327-083 & 64.46 & 22.90 \\
\hline WD 0109-052 & 23.56 & 24.57 & & & 24.98 \\
\hline WD 0135-052 & 27.33 & 1.87 & WD $1527+091$ & 88.86 & 48.40 \\
\hline WD $0148+467$ & 21.92 & 1.95 & WD $1538+269$ & 44.37 & 24.01 \\
\hline WD $0205+250$ & 18.92 & 23.63 & & & 24.97 \\
\hline WD $0227+050$ & 21.46 & 47.40 & WD 1615-154 & 175.50 & 3.67 \\
\hline WD $0232+035$ & 79.67 & 47.45 & & & 21.95 \\
\hline WD $0410+117$ & 33.66 & 23.17 & & & 23.47 \\
\hline \multirow[t]{4}{*}{ WD $0644+375$} & 58.34 & 19.77 & & & 25.62 \\
\hline & & 3.46 & & & 45.42 \\
\hline & & 25.22 & & & 49.09 \\
\hline & & 22.33 & WD $1647+591$ & 71.24 & 3.77 \\
\hline \multirow[t]{5}{*}{ WD $0612+177$} & 70.47 & 20.59 & & & 46.48 \\
\hline & & 2.66 & & & 50.25 \\
\hline & & 22.95 & WD $1919+145$ & 134.98 & 2.25 \\
\hline & & 2.27 & WD $2032+248$ & 74.76 & 1.67 \\
\hline & & 19.93 & & & 47.20 \\
\hline WD $1105-048$ & 62.71 & 23.75 & & & 48.87 \\
\hline \multirow[t]{4}{*}{ WD $1134+300$} & 76.47 & 23.14 & WD 2039-202 & 140.60 & 47.22 \\
\hline & & 20.76 & & & 1.80 \\
\hline & & 3.79 & & & 49.02 \\
\hline & & 21.28 & WD $2117+539$ & 18.62 & 22.63 \\
\hline \multirow[t]{4}{*}{ WD $1143+321$} & 93.71 & 3.85 & WD $2126+734$ & 22.66 & 22.38 \\
\hline & & 22.53 & WD $2149+021$ & 25.79 & 2.70 \\
\hline & & 25.10 & & & 45.64 \\
\hline & & 21.40 & & & 48.34 \\
\hline \multirow[t]{4}{*}{ WD $1254+223$} & 81.93 & 3.55 & WD $2256+249$ & 39.27 & 21.03 \\
\hline & & 26.18 & & & 23.53 \\
\hline & & 22.63 & & & 44.56 \\
\hline & & 21.62 & WD $2309+105$ & 88.76 & 20.73 \\
\hline \multirow[t]{3}{*}{ WD $1314+293$} & 310.89 & 22.18 & WD $2326+734$ & 22.76 & 22.48 \\
\hline & & 23.12 & & & 23.07 \\
\hline & & 45.90 & & & 45.55 \\
\hline
\end{tabular}



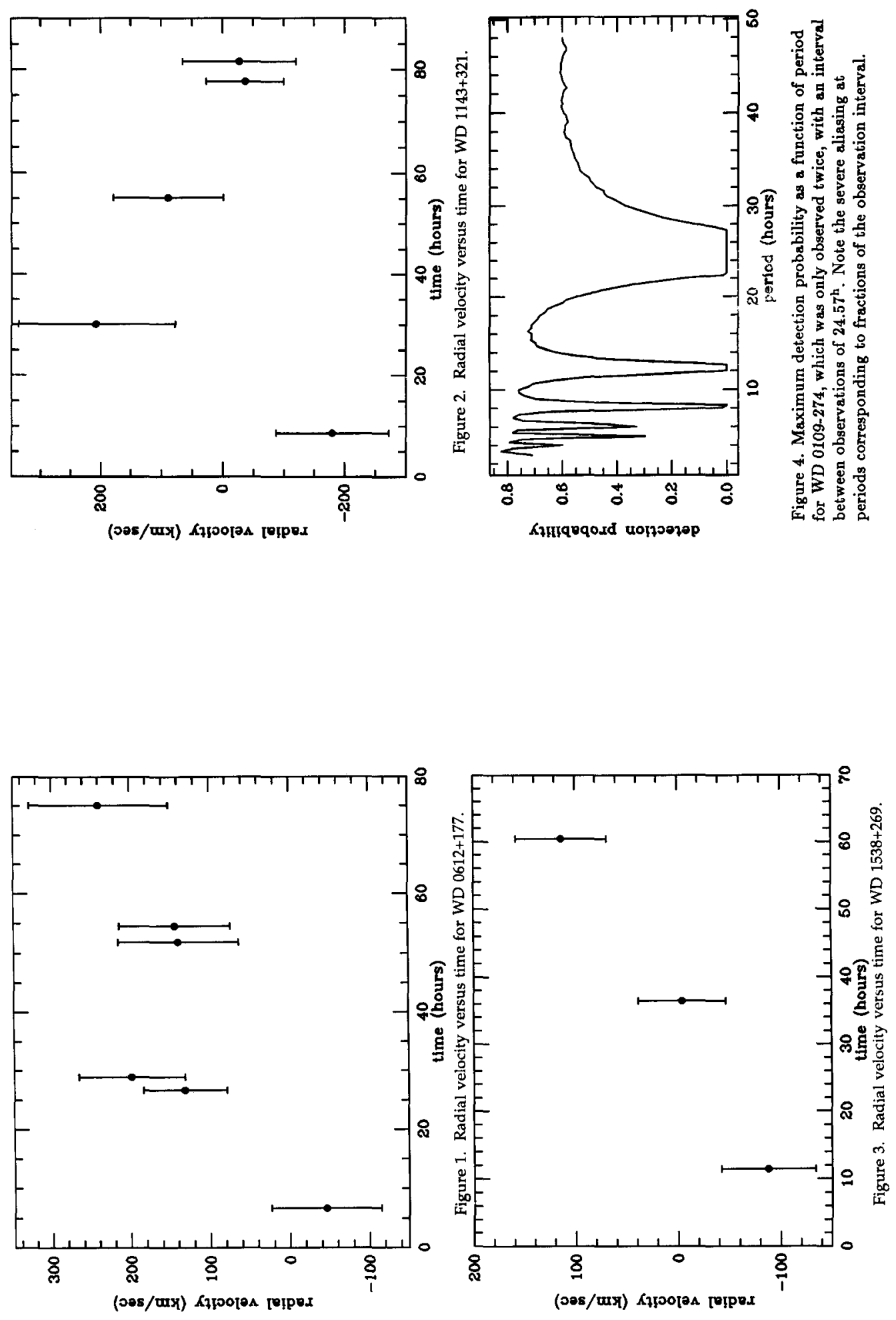
spectra of a helium comparison arc lamp. Each star was observed at least twice, many three or four times, with observations separated by roughly either $3,24,48$, or 72 hours.

The data were reduced using NOAO's Image Reduction and Analysis Facility (IRAF). The spectra were flat-fielded, sky-subtracted, summed down to one dimension and wavelength calibrated using the He arc spectra. They were not flux calibrated. The spectra were fit with a nonlinear least-squares "pseudo" Lorentzian line profile, as described in Saffer, et al. (1988). A velocity shift of at least three times the $1 \sigma$ errors from the fit and the wavelength calibration was considered the minimum believable difference. Three stars, WD $0612+177$, WD 1143+321, and WD1538+239 met this criterion, as shown in Figures 1 through 3.

\section{Discussion}

Although three stars in the sample showed sufficient radial velocity variations to be counted as binaries in the analysis, one cannot therefore immediately calculate a space density of binary white dwarfs. Due to the non-ideal sampling of periods imposed by the intervals between observations, the probability of detecting a binary depends upon its period, dropping to zero when the period is equal to the observation interval. This is shown in Figure 4. Since radial velocity variations could not be detected with $100 \%$ efficiency, any space density calculated directly from these stars plus WD 0135-152 can only be a lower limit, as non-detections in this survey do not necessarily imply single stars. I plan to observe the three stars reported here as candidates, to confirm their radial velocity variability, and to determine their orbits.

\section{References}

Arnett, W.D., Branch, D. and Wheelet, J.C. 1985, Nature , 314, 337.

Greenstein, J. E. 1986, A. J. 92, 867.

Iben, I., Jr. and Tutukov, A. V. 1984, Ap. J. Supp. 54, 335.

McCook, G. P. and Sion, E. M. 1984, A Catalog of Spectroscopically Identified White Dwarfs, (2nd edition, Villanova, Pa., Villanova Press).

Paczynski, B. 1985, in Cataclysmic Variables and Low Mass X-Ray Binaries, Eds. D. Q. Lamb and J. Patterson, (Dordrecht, Reidel), p. 1.

Robinson, E.L and Shafter, A.W. 1987 Ap. J. 322, 296.

Saffer, R. A., Liebert, J. W. and Olszewski, E. W. 1988 Ap. J., in press.

Tornambé, A. and Matteuchi, F. 1986, M.N.R.A.S. , 223, 69.

Webbink, R. F. 1984, Ap. J., 277, 355. 